\title{
Phonon sidebands of localized excitons in molecular crystals with methyl torsions: Hexamethylbenzene*
}

\author{
Steven D. Woodruff, Paras N. Prasad, and Raoul Kopelman \\ Department of Chemistry, The University of Michigan, Ann Arbor, Michigan 48104 \\ (Received 3 December 1973)

\begin{abstract}
Fluorescence and phosphorescence phonon sidebands of isotopic mixed hexamethylbenzene crystals at $2^{\circ} \mathrm{K}$ are presented. The external phonons can be observed separately from the semi-internal (methyl torsion) ones. The nature of the electronic or vibronic state has observable but not drastic effects on the exciton-phonon coupling function. Likewise, the exciton delocalization is of minor importance to the exciton-phonon function in hexamethylbenzene. The coupling between external and internal vibrations is also weak in this system. The exciton-phonon coupling appears comparable for the optical and acoustic phonons in hexamethylbenzene. The phonon sidebands give some of the phonon singularities of the low-temperature crystal.
\end{abstract}

\section{INTRODUCTION}

Singularities of the phonon density of states have been obtained in the past ${ }^{1-3}$ by neutron incoherent scattering (NIS). Recently we suggested ${ }^{4}$ a different method utilizing the phonon sidebands of the guest electronic emission in an isotopic mixed crystal to get such information. It is known ${ }^{5}$ that due to the large neutron scattering cross section of the hydrogen atom, the NIS is heavily biased towards motions with large amplitude at the hydrogen atom. For this reason the NIS spectra ${ }^{1,2}$ of crystals with methyl torsion are dominated by the torsional bands which often obscures the features due to the phonon density of states. The present paper deals with the investigation of such a system by our isotopic mixed crystal method and discusses its relative merits.

The Raman spectra ${ }^{6,7}$ of the isotopic mixed crystals show that the methyl torsions (which we have called ${ }^{8}$ semi-internal modes) have a large deuteration shift compared to their exciton bandwidth and are always in the separated band limit. ${ }^{B}$ Furthermore, the $\mathbf{k}=0$ torsional vibration frequencies of the protoguest have been found to be located above the phonon bands of the crystal ${ }^{6,7}$ so that delocalization of the torsional vibration through phonon coupling is expected to be small. For both these reasons, the torsions of the guest are expected to be significantly localized at the guest site and should appear as sharp vibronic transitions that do not interfere much with the broad structured phonon sideband, the latter giving the singularities of the external phonon density of states. Also, for the same reason, the torsional bands of the perdeuterohost should have only small amplitudes at the guest site and the transitions of two-particle nature (guest electronic state emitting into the host torsional band) will be weak. In view of all these considerations it appears that for crystals with methyl torsions our method should provide a complimentary if not better mapping of the phonon density of states compared to the one obtained by the NIS method.

We present here phonon sideband studies in both fluorescence and phosphorescence of isotopic mixed hexamethylbenzene (HMB) crystals at $2^{\circ} \mathrm{K}$. The hexamethylbenzene crystal is selected as it has the added advantage of having only one molecule per unit cell broth in its room temperature phase and in its low temperature phase (see below) investigated here. The phonon sidebands of the fluorescence $0-0$, the $450 \mathrm{~cm}^{-1}$ vibronic fluorescence origin (nontotally symmetric) and the phosphorescence 0- 0 are compared so as to investigate the dependence of the exciton-phonon coupling function on the molecular nature of the transition. A concentration dependence study of the phonon sideband provides information about the effect of guest excitation delocalization on the phonon sideband and also helps in identifying trap emissions. A qualitative estimate of the coupling between the external vibration and the internal vibration ${ }^{9}$ is made on the basis of the comparison between the phonon sidebands of the 0-0 and its vibronic band.

Finally a comparison of the crystal Raman spectrum? with the phonon sideband of the electronic emission permits us to comment on the relative strengths of coupling of the localized exciton (the guest excitation) with optical and acoustic phonons.

\section{EXPERIMENTAL}

HMB- $d_{18}$ obtained from Merck, Sharp, and Dohme with the quoted purity of $99 \%$ atom deuterium was used without further purification. HMB- $h_{18}$ from Aldrich was purified by recrystallization from hot ethanol. Isotopic mixed crystals of $0.6 \%, 3 \%$, and $10 \% \mathrm{HMB}-h_{18}$ in HMB- $d_{18}$ were grown from a benzene solution layer over water. The crystals thus obtained were $10-100 \mu$ thick. The crystal was mounted on a sample holder between quartz plates separated by a silver foil gasket to reduce physical strain. The HMB spectrum was found to be highly sensitive to strain. The crystal sample was immersed into supercooled liquid helium $\left(\sim 2{ }^{\circ} \mathrm{K}\right)$ inside a cryostat with no liquid nitrogen in the optical path. The excitation was provided by $90^{\circ}$ illumination with a 1600 watt high pressure xenon lamp using a Jarrel-Ash 0.25 $\mathrm{m}$ monochromator and a $15 \mathrm{~cm}$ water cell as filters. The spectra were taken photographically on a Jarrel-Ash $1 \mathrm{~m}$ double Czerny-Turner spectrograph-spectrometer. The effective resolution was $1 \mathrm{~cm}^{-1}$. We note that we independently checked our crystals, by taking Raman spectra in situ, to make sure that they consisted of the low temperature phase only. ${ }^{7}$ 


\section{RESULTS}

The results of the fluorescence from HMB- $h_{18}$ at three different concentrations in HMB- $d_{18}$ host are compared in Fig. 1. The figure covers the region up to about 380 $\mathrm{cm}^{-1}$ from the fluorescence origin $(0-0)$. In the spectrum from the $0.6 \%$ mixed crystal the entire guest emission comes from the monomer level of the guest. However, we also see an emission band at $104 \mathrm{~cm}^{-1}$ on the higher energy side of the $0-0$. This is due to a trap in the HMB- $d_{18}$ host (it gets weaker in crystals with higher guest concentration and stronger with lower concentration). The lower energy side spectral region (the sideband) is rich in structure. In the $3 \%$ mixed crystal spectrum, the guest emission is somewhat broad, possibly because some emission comes from the dimer level. The bands around $220 \mathrm{~cm}^{-1}$ and at $330 \mathrm{~cm}^{-1}$ get weaker which makes us believe that they are built on the 104 $\mathrm{cm}^{-1}$ trap origin. In the $10 \%$ mixed crystal the emission is predominantly from the dimer. guest level, though some emission is still coming from the monomer (as can be seen by the asymmetry on the $360 \mathrm{~cm}^{-1}$, and other

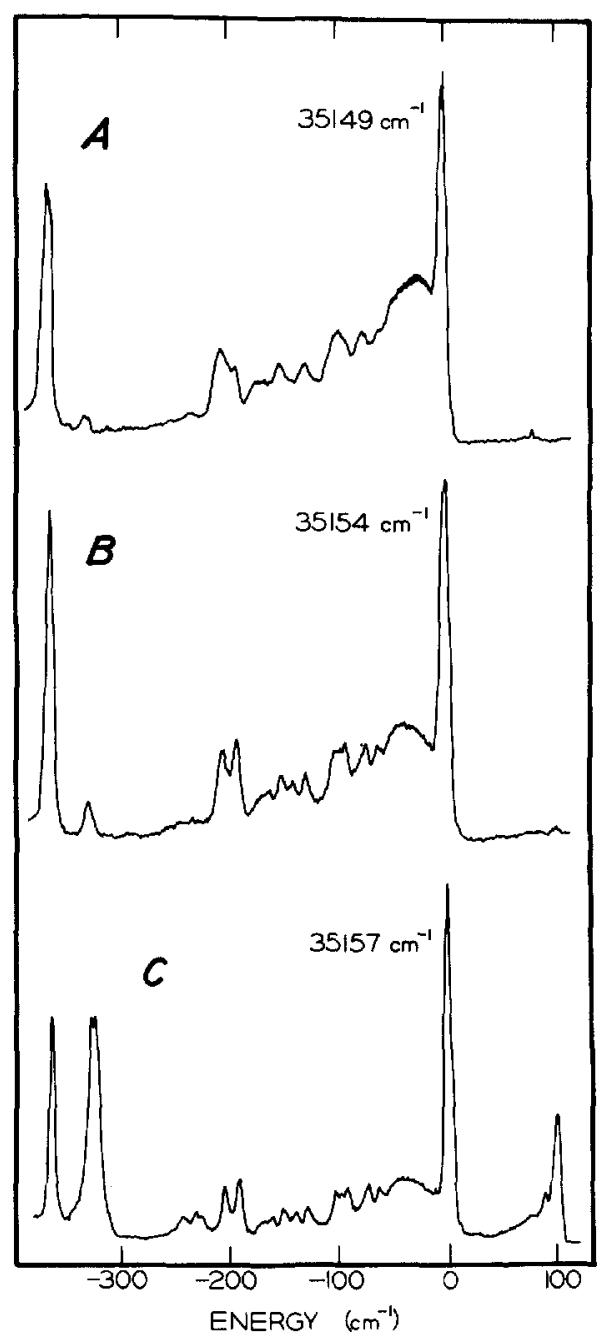

FIG. 1. Densitometer tracings of the $2^{\circ} \mathrm{K}$ fluorescence from isotopic mixed hexamethylbenzene crystals of three different concentrations. $A$ is the spectrum of $10 \% \mathrm{HMB}-h_{18}$ in $\mathrm{HMB}-d_{18}$ mixed crystal. $B$ and $C$, respectively, are the spectra of crystals with $3 \%$ and $0.6 \% \mathrm{HMB}-h_{18}$ in $\mathrm{HMB}-d_{18}$.

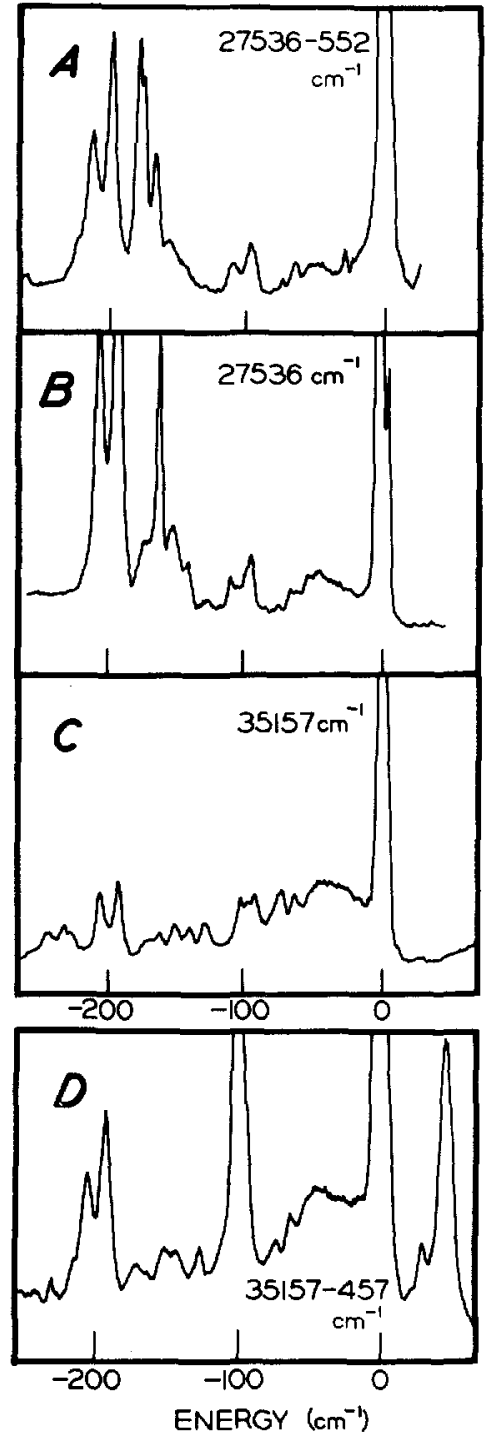

FIG. 2. Comparison of the sidebands of the fluorescence $0-0$, the $457 \mathrm{~cm}^{-1}$ fluorescence vibronic origin, the phosphorescence $0-0$, and the $552 \mathrm{~cm}^{-1}$ phosphorescence vibronic band. All these spectra are taken at $2^{\circ} \mathrm{K}$ on a crystal of composition $0.6 \%$ HMB$h_{18}$ in $\mathrm{HMB}-d_{18}$ as a host. $B$ and $A$, respectively, show the sidebands of the fluorescence $0-0$ and the $457 \mathrm{~cm}^{-1}$ fluorescence vibronic origin, while $C$ and $D$ correspond, respectively, to those of the phosphorescence $0-0$ and the $552 \mathrm{~cm}^{-1}$ phosphorescence vibronic band.

vibronic bands). We see that except for some broadening and small changes in the intensity pattern the sideband is similar to that of the monomer emission.

Figure 2 compares the sidebands of the fluorescence $0-0$, the $457 \mathrm{~cm}^{-1}$ fluorescence vibronic band, the phosphorescence $0-0$ and the $552 \mathrm{~cm}^{-1}$ phosphorescence vibronic band, all taken at $2^{\circ} \mathrm{K}$. The transition at about $95 \mathrm{~cm}^{-1}$ on the lower energy side of the $457 \mathrm{~cm}^{-1}$ fluorescence vibronic band is due to the $552 \mathrm{~cm}^{-1}$ vibration. It can be seen that the sidebands in all these spectra show great similarity. However, there are some apparent differences, especially in the region above $120 \mathrm{~cm}^{-1}$. In the phosphorescence we see a rather strong peak at 161 $\mathrm{cm}^{-1}$ whereas in the fluorescence there is only a weak peak at this frequency. We also have done a concentra- 
tion dependence study on the phosphorescence which shows that the sideband is the same for both $3 \%$ and $0.6 \%$ mixed crystal phosphorescences. The peak at 180 $\mathrm{cm}^{-1}$ in the sideband of the $552 \mathrm{~cm}^{-1}$ phosphorescence vibronic band is, most probably, due to another vibronic band.

\section{DISCUSSION}

Though the precise crystal structure of HMB phase III is not known, our spectroscopic results ${ }^{7}$ provide support for a structure with one centrosymmetric molecule per unit cell and the effective site symmetry seems to be as high as $D_{3 d}$ (it contains at least $C_{3}$ and $C_{i}$, i. e., $S_{6}$ ). ${ }^{10}$ In such a crystal there are three optical phonon branches whose $\mathbf{q}=0$ components have $g$ character and are derived from the molecular librations $\left(R_{x}, R_{y}\right.$, and $\left.R_{z}\right)$. In the $\mathrm{D}_{3 d}$ site symmetry only the degenerate librations $R_{x}$ and $R_{y}$ are expected to show up in the Raman spectrum of the HMB crystal in the low temperature phase. This band for $\mathrm{HMB}-d_{18}$ is at $\sim 100 \mathrm{~cm}^{-1}$. The forbidden $R_{z}$ libration is expected to be below $100 \mathrm{~cm}^{-1}$ (around $60 \mathrm{~cm}^{-1}$ ). ${ }^{11}$ On this basis we attribute the sideband region (0-120 $\mathrm{cm}^{-1}$ ) of the emission to be due to one-phonon transitions, both optical and acoustical. We tend to think that the structure in the sideband region above $120 \mathrm{~cm}^{-1}$ is not due to two or higher phonon processes. This is based on the following grounds: (i) The intensity in the phonon sideband is small compared to that of the corresponding zero-phonon transition. This can be seen on the vibronic bands of both fluorescence and phosphorescence while for the electronic band it can be seen more clearly in the phosphorescence where the zero-phonon transition is not reabsorbed. The above implies small exciton-phonon coupling ${ }^{12}$ which makes two or multiphonon processes improbable. (ii) The features above 120 $\mathrm{cm}^{-1}$ decrease in peak intensity as the guest concentration increases. ${ }^{13}$ As an increase in guest concentration should not change the phonon features of the crystals (the phonons are in the amalgamation limit ${ }^{6,8}$ ), the features above $120 \mathrm{~cm}^{-1}$ do not seem to be due to external phonons. The possibility of some of them being due to two-phonon processes, however, cannot be completely ruled out. On the other hand, one methyl torsion of HMB- $h_{18}$ has been identified by Raman spectra ${ }^{7}$ to be at $165 \mathrm{~cm}^{-1}$. The corresponding torsional band $d^{7}$ of $\mathrm{HMB}-d_{18}$ is at $125 \mathrm{~cm}^{-1}$. Furthermore, as our HMB- $d_{18}$ has only $99 \%$ d atom purity, there is a significant isotopic impurity distribution in the HMB- $d_{18}$ host (e.g., about $16 \%$ mole HMB- $d_{17} h$, etc.). These impurities will give rise to quasi-extended torsional bands between $165 \mathrm{~cm}^{-1}$ and $125 \mathrm{~cm}^{-1}$. The features in the sideband region above $120 \mathrm{~cm}^{-1}$ might be due to these bands. ${ }^{14}$ We note, however, that the identification of the methyl torsional band in the emission spectra, the nature of its coupling with the electronic transition and the assigniment of other features above $120 \mathrm{~cm}^{-1}$ will be discussed in a separate publication. In the present paper we focus our attention on the one-phonon transition region, $0-120 \mathrm{~cm}^{-1}$, and only the spectral features of this region will be called, collectively, the one phonon sideband (see Fig. 3). We have shown ${ }^{4}$ that the intensity distribution for the onephonon sideband for the state $f$ of the guest is given as

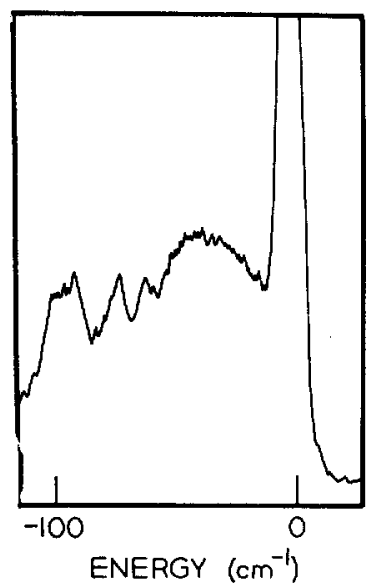

FIG. 3. External one-phonon sideband ("singularity mapping") of HMB, phase III [enlarged portion of Fig. $1(b)]$.

$$
I_{1}^{f}(\omega)=(12 N)^{-1} \sum_{S, q} C_{S, q}^{f} \delta\left(\omega-\omega_{S, q}\right) .
$$

As long as the $C_{s, q}^{f}$ do not have any singularities, $I_{1}^{f}(\omega)$ would map the singularities of the phonon density of states. Possible factors determining the exciton-phonon coupling function $C_{S, Q}^{f}$ are as follows: the molecular nature of the guest excitation, the extent of its delocalization, the phonon wave vector $q$, and the polarization $S$. In the HMB crystal the singlet state $\left({ }^{1} B_{2 u}\right.$ in $\left.\mathrm{D}_{6 h}\right)$ and the triplet state $\left({ }^{3} B_{1 u}\right)$ are of different orbital symmetry, and the $457 \mathrm{~cm}^{-1}$ vibronic band of the fluorescence, corresponding to an $E_{25}$ mode of benzene, is a Herzberg-Teller induced vibronic origin, essentially borrowing intensity from the allowed $\left({ }^{1} E_{1 u}\right)$ state. The similarity among the phonon sidebands of these three emissions, coming from excited states of effectively different electronic symmetry, indicates that the coupling function $C_{S, q}^{f}$ is not very sensitive to the molecular nature of the guest excited state. Furthermore, as the effective site symmetry $\left(D_{3 d}\right)$ in these crystals is as high or almost as high as the molecular symmetry ${ }^{7}$ the above observation also indicates that the coupling function $C_{S, q}^{f}$ is not selective to a particular phonon of well defined polarization. The fact that the phonon sideband of the dimer emission resembles that of the monomer emission indicates that the effect of delocalization of the guest electronic state on the coupling function $C_{S, q}^{f}$ is also small.

The similarity of the phonon sidebands built on various vibronic bands (whether totally symmetric or not) with those built on the corresponding pure electronic emissions suggests that the coupling between the external vibration and the internal vibration is small and the coupling function $C_{S, q}^{f}$ is not very sensitive to the internal vibrational states. This is also supported by our published ${ }^{7}$ and unpublished Raman data where these bands do not show any significant effect of coupling between the internal vibrations and the extermal vibrations. The manifestations of such an interaction would be a temperature dependence of the vibrational frequency, ${ }^{9}$ large temperature broadening, and phonon sidebands built on these vibrational transitions in the Raman spectra. No such effects were observed.

The insensitivity of the coupling function $C_{S, q}^{f}$ to its 
possible variables discussed above leads us to believe that this function does not have any singularities and thus the phonon sideband is essentially mapping the significant singularities of the phonon density of states in the ground state of the crystal $^{15}$ (see Fig. 3).

The hexamethylbenzene crystal has also been investigated by the NIS method. ${ }^{1}$ Comparing the NIS result with that obtained by our method, the relative merits of our method for molecular crystals with methyl torsion can easily be seen. In the low temperature phase NIS spectrum, the band due to the methyl torsions $\left(135 \mathrm{~cm}^{-1}\right.$ peak at $100{ }^{\circ} \mathrm{K}$ ) dominates and obscures any phonon features above $60 \mathrm{~cm}^{-1}$. These features can clearly be seen in our spectra. In principle, NIS should be especially suited for HMB. As the methyl torsions here do not overlap with the external phonons, the bands due to methyl torsions and those due to external phonons should be well separated. However, multiphonon contributions do increase the overlap in the NIS spectra, thus washing out many structural features of the external phonon bands. We would like to point out that in a number of crystals with methyl torsions (e.g., durene $\mathrm{f}^{6}$ ), the separation between the lowest methyl torsional band and the highest external phonon band is either small, or they actually overlap. In such cases, even in the absence of the multiphonon process, NIS would not be able to provide detailed information about the phonon density of states. On the other hand, in our method the methyl torsions would cause little or no interference with the external phonon sideband. We attribute the HMB features above $60 \mathrm{~cm}^{-1}$ to the optical phonons. The structure (peaks) near $70 \mathrm{~cm}^{-1}\left(62 \mathrm{~cm}^{-1}\right.$ and $\left.72 \mathrm{~cm}^{-1}\right)$ seems to be derived from the optical phonon branch originating from the $R_{z}$ libration. The peaks near $95 \mathrm{~cm}^{-1}(102,98$, and $\left.91 \mathrm{~cm}^{-1}\right)$ are most probably due to the optical phonon branches which belong to the $R_{x}$ and $R_{y}$ librations. The broad band that peaks around $40 \mathrm{~cm}^{-1}$ is attributed to the acoustic phonons. ${ }^{16}$ It can be seen that the couplings (given by the spectral intensities) with acoustic phonons and optical phonons appear to be of comparable magnitude, provided that the $R_{z}$ libration is not responsible for the intensity in the low energy region.

Note added in proof: Very recently P. A. Reynolds [Chem. Phys. Lett. 22, 177(1973)] has claimed that the phonon sidebands on excitons in molecular crystals measure a function related to some of the Brillouin Zone Center translational mode frequencies. He based his claim on a theoretical model that assumes a spherical symmetry for both the electron density and the $\Pi-\Pi^{*}$ transition density, combined with his interpretation of experimental data on neat crystals of pyrazine and paradichlorobenzene and isotopic mixed crystals of naphthalene (disagreeing with our own interpretation of our experimental data ${ }^{4}$ ). Hexamethylbenzene is certainly not less spherical than the above molecules. On the other hand, in this crystal having only one molecule per primitive unit cell, translational phonon sidebands at or close to $q=0$ will have zero or close to zero frequencies, in sharp contrast with our observed sideband that stretches over more than $100 \mathrm{~cm}^{-1}$ (as do the rotational Raman band $\left.s^{7}\right)$. We therefore conclude that the above hexamethylbenzene data sharply contradict Reynolds' approach.

\section{CONCLUSIONS}

(1) Our method of identifying phonon singularities is less affected by the presence of methyl torsions, compared with neutron incoherent scattering.

(2) For the low temperature crystal phase of hexamethylbenzene our conclusions are as follows: (a) The electronic exciton-phonon coupling function seems to be little perturbed by delocalization. Furthermore, it is not very sensitive to the nature of the three lowest excited electronic states, thereby enabling one to use the method of the localized exciton sideband for the determination of phonon singularities. (b) The coupling between external and internal vibrations is weak. (c) The exciton-to-external-phonon coupling appears to be comparable for optical and acoustical phonons.

*Supported by NSF Grant No. GH-32578 and NIH Grant No. NS08116.

${ }^{1}$ J. J. Rush and T. I. Taylor, J. Chem. Phys. 44, 2749 (1966).

${ }^{2}$ J. J. Rush, J. Chem. Phys. 47, 3936 (1967).

${ }^{3}$ P. A. Reynolds, J. K. Kjems, and J. W. White, J. Chem. Phys. 56, 2928 (1972).

${ }^{4}$ R. Kopelman, F. W. Ochs, and P. N. Prasad, J. Chem. Phys. 57, 5409 (1972).

${ }^{5} \mathrm{H}$. Boutin and S. Yip, Molecular Spectroscopy with Neutrons (M. I. T. press, Cambridge, MA, 1968).

${ }^{6}$ P. N. Prasad and R. Kopelman, J. Chem. Phys. 58, 126 (1973).

${ }^{7}$ P. N. Prasad, S. D. Woodruff, and R. Kopelman, Chem. Phys. 1, 173 (1973).

${ }^{8}$ P. N. Prasad and R. Kopelman, Chem. Phys. Lett. 21, 505 (1973).

${ }^{9}$ P. N. Prasad and R. Kopelman, J. Chem. Phys. 58, 5704 (1973).

${ }^{10} \mathrm{Dr}$. J. E. Edmonds (private communication) has informed us that the neutron diffraction studies of the low temperature phase suggest a trigonal crystal form with one molecule per primitive cell and the unit cell containing symmetry elements $\mathrm{C}_{i}$ and $\mathrm{C}_{3}$ (nearly hexagonal symmetry). Very recently Bertinelli and Stremmenos [J. Chem. Soc. Faraday II, 69, 889 (1973)] agreed with this structure. Even though our ${ }^{7}$ interpretation of the Raman spectra disagrees sharply with theirs, our interpretation is also consistent with $\mathbf{S}_{6}$ symmetry (with the effective phonon force field being close to a $\mathbf{D}_{3 d}$ or even $\mathbf{D}_{6 h}$ symmetry).

${ }^{11}$ This is derived from the fact that the $R_{z}$ libration involves the lowest moment of inertia. Also, as this motion has a nearly sixfold barrier, its force constant is expected to be the softest of all the librations. Furthermore, for the high temperature triclinic form where a nearly hexagonal arrangement is seen in the plane 001 (the low temperature and high temperature crystal forms probably only differ in the packing of these planes), the calculation based on root mean square amplitude as derived from the neutron diffraction studies [W. C. Hamilton, J. E. Edmonds, A. Trippe, and J. J. Rush, Discuss. Faraday Soc. $48,192(1969)$ ] shows that the $R_{z}$ libration should be the lowest frequency libration. However, it is possible that this simple picture is not valid, and the $R_{z}$ libration is not below the $R_{x}$ and $R_{y}$ librations but rather lies in the region above $100 \mathrm{~cm}^{-1}$ for the HMB-d $d_{18}$ crystal. Even so, this would not affect our basic conclusions given below.

${ }^{12}$ R. M. Hochstrasser and P. N. Prasad, J. Chem. Phys. 56, 2814 (1972).

${ }^{13}$ We have done a concentration dependence study on the fluorescence going below $0.6 \%$ and above $10 \%$ of $\mathrm{HMB}-h_{18}$. 
${ }^{14} \mathrm{Finally}$, it is also possible that the $R_{z}$ libration lies in this region (see Ref. 11). In such a situation some of the features above $120 \mathrm{~cm}^{-1}$ would be derived from the one phonon transitions of the $R_{z}$ librational branch. The doublet feature around $200 \mathrm{~cm}^{-1}$ is due to internal vibrations.

${ }^{15}$ Even if the individual coupling function $C_{S, q}^{f}$ has an oscillatory behavior when plotted against $q, \mathrm{Eq}$. (1) contains a summation of such functions at each energy point (with a given $\omega$ whose width is defined by the experimental resolution). We believe that the summation over such functions will average out such oscillations, resulting in an effective smoothing of these features, except when directly related to singularities.

${ }^{16} \mathrm{~A}$ comparison of the above frequencies with those of the neat crystal sidebands will be given in a future publication. 